Report

\title{
An Analysis of Severe Coal Mine Trauma Curing According to 144 Examples
}

\author{
Sun Bingfeng ${ }^{1, ~}{ }^{\text {, }}$, Fan Shifeng ${ }^{1}$, Fu Qiang ${ }^{1}$, Gao Yuan ${ }^{1}$, Wang Wei ${ }^{1}$, Ye Chunyong ${ }^{2}$, Hu Guolun ${ }^{3}$, \\ Wang Xinpan ${ }^{4}$, Chen Hongyan \\ ${ }^{1}$ Department of Shuikuang Holding Limited Liability Company General Hospital, Liupanshui City, China \\ ${ }^{2}$ Department of Liuzhi Special District People's Hospital, Liupanshui City, China \\ ${ }^{3}$ Department of Panjiang Investment Holding Corporation General Hospital, Liupanshui City, China \\ ${ }^{4}$ Department of Pan County People's Hospital, General Hospital, Liupanshui City, China \\ ${ }^{5}$ Department of Liupanshui City Secondary People's Hospital, General Hospital, Liupanshui City, China \\ Email address: \\ sbf8179875@126.com (Sun Bingfeng) \\ ${ }^{*}$ Corresponding author
}

\section{To cite this article:}

Sun Bingfeng, Fan Shifeng, Fu Qiang, Gao Yuan, Wang Wei, Ye Chunyong, Hu Guolun, Wang Xinpan, Chen Hongyan. An Analysis of Severe Coal Mine Trauma Curing According to 144 Examples. European Journal of Clinical and Biomedical Sciences.

Vol. 5, No. 3, 2019, pp. 43-45. doi: 10.11648/j.ejcbs.20190503.11

Received: June 3, 2019; Accepted: July 11, 2019; Published: July 23, 2019

\begin{abstract}
Introduction: improve the level of curing severe coal mine trauma patients, find out the regulations and deficiencies of coal mine severe trauma treatment Method: summarize and analyze 144 case data of severe coal mine trauma patients from 5 hospitals in Liupanshui City from March 2014 to March 2017 Result: 144 severe coal mine trauma patients are all males and their average age is 40.92 years old. Most of them hospitalize in the ICU (admission from April to June, about $29.17 \%$ ) while fewest of them (admission from July to September, about 17.26\%). Most patients hospitalize in the ICU about 0.85 days after the severe coal mine trauma happens and the average treatment time of ICU is 15.15 days. Patient source comes most from the people of emergency (94.44\%), fewest from the people who transferred after experienced treatment in other hospital. The average score of SOFA evaluation is 4.1 while the average score in APACHE II evaluation is 16.2. Most kind of work which trauma takes place easily is coal mining (74.31\%); other jobs which trauma also happens are driving (7.64\%), ventilation $(8.33 \%)$, transportation $(6.94 \%)$, electromechanics $(1.39 \%)$. The main reason of trauma is roof failure $(41.67 \%)$, and there're other reasons like harvesters extrusion (36.11\%), falling (7.64\%), electromechanics explosion (7.64\%), high gas $(5.56 \%)$. The main hurt organs are craniocerebal, lung, abdomen, limbs, centrum, maxillofacial. The implementation rate of tertiary rescue is $79.86 \%$. Finally, 135 people survive after treatment while 9 people die. Conclusion: The treatment of severe coal mine trauma has its own regulation and characteristic, attaching high importance to the coal mine security, tertiary rescue and trauma curing is benefit to improve the success rate of curing severe coal mine trauma.
\end{abstract}

Keywords: Coal Mine, Trauma, Severe, Curing, Liupanshui City

\section{Data and Information}

General data: This subject collect 144 case data from March 2014 to March 2017. They're all males from 28 years old to 54 years old, while the average age is 40.92 years old. Admission in ICU: 38 cases from January to March, 42 cases from April to June, 25 cases from July to September, 39 cases from October to December. Most patients hospitalize in the ICU about 0.85 days after the severe coal mine trauma happens and the average treatment time of ICU is 15.15 days. Patient source: 136 cases of emergency, 6 cases of inside transferring, 2 cases of transferring after experienced treatment in other hospital. The average score of SOFA is 4.1 while the average score in APACHE II is 16.2. Kind of work with trauma: 136 cases of coal mining, 12 cases of driving, 2 
cases of electromechanics, 10 cases of transportation, 11 cases of ventilation, 2 cases of other works. Reasons of trauma: 60 cases of roof failure, 52 cases of harvesters extrusion, 11 cases of falling, 11 cases of electromechanics explosion, 8 cases of high gas, 2 cases of other situation. Main hurt organs: 58 cases of craniocerebal, 29 cases of lung, 10 cases of abdomen, 23 cases of limbs, 22 cases of centrum, 2 cases of maxillofacial. People who accept tertiary rescue is 115 .

Method: 5 state-owned hospitals in the coal mine production area in Liupanshui City provide the original data, and all the patients will be mainly treated by the ICU. We adopt APACHE II evaluation and SOFA evaluation as the trauma judgment standard. The tertiary rescue standard adopts the General hospital- mine hospital- wellhead/ downhole rescue system. We will do retrospective analysis about the patient's age, trauma rescue time, treatment source, type of work, main trauma reason, main hurt organ, implementation rate of tertiary rescue and final outcome.

Statistics: We adopt SPSS 17.0 software to do statistic analysis, we will use the measurement data to do the Student's t test, if the $\mathrm{p}$ value is below 0.05 , these data will be significant.

\section{Findings}

135 people survive (including 106 people who are cured by tertiary rescue), 9 people die. The contrast of treatment between the survivors and deaths from severe coal mine trauma and the deaths is shown in the following chart:

Table 1. The contrast of treatment between the survivors and deaths from severe coal mine trauma and the deaths.

\begin{tabular}{|c|c|c|c|c|c|c|}
\hline Group & Number & Age/years & Admission to the ICU after hurt/day & ICU treatment/day & SOFA/score & APACHE II/score \\
\hline Survivors & 135 & $40.02-40.66$ & $0.44-0.68$ & $8.16-8.24$ & $4.44-4.56$ & $17.95-18.29$ \\
\hline Deaths & 9 & $45.27-45.73$ & $0.87-0.95$ & $23.73-24.77$ & $5.69-7.11$ & $22.43-27.07$ \\
\hline
\end{tabular}

PS: Comparing with the deaths, the p value is below 0.01 .

\section{Discussion}

The Liupanshui City's every governments pay high attention to the coal production security. During the twelfth Five-Year period, the whole city has invested to build a "safe cloud" data monitoring system and improve the wellhead and downhole telecommunication and monitoring system whose core is a safety production scheduling command center which includes positioning, video monitoring, gas and carbon monoxide on-time monitoring; besides, these systems can monitor all the 209 mining production in the whole city online. Liupanshui District's water mine and the Panjiang mine secure team all provide resource to the country-level rescue team, which means they will support the team when rescuing the coal mine patients and operating the tertiary rescue standard at the first time. Therefore, the saving rate can up to $79.86 \%$. Since 2008 , ICU has been independent as a secondary discipline, therefore it brings convenience for Liupanshui City's severe coal mine trauma patients can be rescued intensively in every coal mine hospitals and the success rate of this treatment can achieve 93.75\%. During the twelfth Five-Year period, the death rate of whole city's billion GDP project is $4 \%$ and the number is lower than the national level and the provincial level [1]. In the recent 3 years, few disaster, death and gas explosion take place in Liupanshui City's coal mine trauma, but most are roof failure, harvesters extrusion and falling and they're obviously not same as the country investigation about gas explosion and high gas [2].

Liupanshui City isan important base for national plan of "West to Eat Power Transmission Project", most of them are thermal power. Each year's flood season peak of the Three Gorges's hydroelectric generation and the demand of thermal power is fewer. The driving task is slower and the workload is less; that's why from July to September, the trauma happens the fewest all the year round. However, middle-aged miners are special workers for their knowledge and social fitness abilities are not very well, so they have to driving mine and that's why severe coal mine trauma patients' average age is 40.92 years old and those people have high risks.

Craniocerebal injury happens most in the severe coal mine trauma and our conclusion is as the same as peers' [2]. because of the speciality of coal driving environment, craniocerebal injury happens easily. At that time, the impact force is very high and the wounded area is large; chronic axonal injury, generalized cerebral contusion and delayed intracrnial hematoma are the characteristics of severe coal mine trauma. When the craniocerebalstem is injured, the craniocerebalstem reflection keeps decreasing, the GCS becomes lower and predicts patients can die in a short time [3]. For severe coal mine trauma patients, cerebal compression should be removed as soon as possible, which means in the early time, open your craniocerebal and clean the intracranial hematoma, all this should be done in "one golden hour". during the treatment, vital signs, changes on pupils and CT review should be the focus. Due to the limitation of coal mine trauma area and coal mine worker's knowledge, the behaviour of taking injured people to hospital is not very useful; after sending to the hospital, the craniocerebal CT is valued but the neck examination and cervical spine imaging are always ignored which leads to the review. According to the statistics, the rate craniocerebal injury which leads to mislead diagnosis ranges from $2.8 \%$ $6.9 \%[1,2]$; therefore, when rescuing the craniocerebal injures at the first time to protect the cervical vertebra and avoid diagnosis.

Respiratory system is the secondary damage organ. Miners' lung injury usually extensive contusion of both lungs, when the gas is high or explodes, the lung will suffer 
from inhalation injury. Sharp knocks on the lung will lead to alveolar rupture, pulmonary hemorrhage, pulmonary edema and emphysema, and pleural effusion [1]; some serious contusion can cause death with the help of hyoxemia and ARDS [4]. In the early period, if patients can antagonize excessive inflammation in the alveoli, restricted fluid volume resuscitation [4], keep airways open and maintain the integrity of the chest gallery; he or she will recover faster. Serious flail chest should be fixed at the early operation. After lung injury, from 48 hours to 72 hours, pulmonary contusion will exudate in a peak, so patients have to pay attention to the CT review of lung and signs. In the early period of pulmonary atelectasis, medical ways are needed to use in clinic. At present, the blood observation can simultaneously observe the change of $\mathrm{COHb}$, and we can judge when and how to use HPO method to treat patients who experience high gas or gas explosion [2].

Restricted by the present situation, our research data can't explain all the reason of dead people in the coal mine accident, so the findings will be influenced. But Liupanshui City's severe coal mine trauma has its own regulation and characteristics, the government and enterprises will continue attaching high importance to the coal mine safety, tertiary rescue; to build the ICU medicine as the comprehensive platform for medical rescuing and improve the rate of severe coal mine trauma.

\section{References}

[1] Wang Fei, Huo Yingxu. Liupanshui City's Big Data Safety Case Analysis [Z], 2017: 193.
[2] Fan Yaqing. Our Country's Coal Mine Disaster Characteristic and Regulation Analysis from 2012 to 2015 [J]. Coal, 2016, 25 (5): 16-18.

[3] Xu Jianqiang, Huang Xin, Sun Yongdong. Epidemic Disease Study of Miners Craniocerebral Trauma [J]. China's Coal Industry Medical Magazine, 2010, 13 (1): 115-117.

[4] Li Shijun, Zeng Jun, Liu Fajian. Severe craniocerebal Trauma Patient's Prognostic Factor Analysis after Operation [J], Sichuan Medical, 2013, 34 (3), 294-297.

[5] Zhou Xiyuan, Liu Kaijun. Review of the Treatment of Craniocerebral Injury Cervical Spinal Cord Injury [J]. Traumatology Departmet Magazine, 2010, 12 (6): 504-507.

[6] Tian Hengli, Xu Tao, Gu Bingxian. Clinic Characteristics of Craniocervical Injury, Chin J Nerv Ment Dis, 2004, 30 (6): 463-464.

[7] Hu Bing, Meng Weizheng, Ren Wenjie. Clinic Characteristics of Lung Detonation Injury which Caused by Gas Explosion Burns [J]. Chinese Journal of Industrial Hygiene and Occupational Diseases, 2008, 26 (2): 126-127.

[8] PERNA V, MORERA R. Prognostic Factors in Chest Traumas: A Prospective Study of 500 Patients [J]. Cir Esp, 2010, 87 (3): 165-170.

[9] Shu Zhenyun, Chen He, Liao Jinping. Different Liquid Resuscitation Methods to Severe Chest Trauma Combined with Shock Victims ARDS and Improvement of Prognosis [J]. Hebei Medicine, 2016, 22 (9): 1435-1438.

[10] Zhang Suqing, Liu Deli, Han Baode. 30 Cases of Hyperbaric Oxygen Therapy for Acute Gas Asphyxia [J]. Ningxia Medical Magazine, 2011, 33 (1): 76-77. 